\title{
Relationships of affective temperament ratings to diagnosis and morbidity measures in major affective disorders
}

\section{Research Article}

Cite this article: Miola A, Baldessarini RJ, Pinna M, Tondo L (2021). Relationships of affective temperament ratings to diagnosis and morbidity measures in major affective disorders. European Psychiatry, 64(1), e74, 1-7 https://doi.org/10.1192/j.eurpsy.2021.2252

Received: 01 October 2021

Revised: 10 November 2021

Accepted: 13 November 2021

Keywords:

Affective temperaments; bipolar disorders; diagnosis; major depression; TEMPS-A

Author for correspondence:

*Leonardo Tondo,

E-mail: Ltondo@aol.com (c) The Author(s), 2021. Published by Cambridge University Press on behalf of the European Psychiatric Association. This is an Open Access article, distributed under the terms of the Creative Commons Attribution licence (http:// creativecommons.org/licenses/by/4.0), which permits unrestricted re-use, distribution and reproduction, provided the original article is properly cited.

EUROPEAN PSYCHIATRIC ASSOCIATION

\author{
Alessandro Miola ${ }^{1}$, Ross J. Baldessarini ${ }^{2,3}$, Marco Pinna ${ }^{4}$ (1) and \\ Leonardo Tondo ${ }^{2,3,4 \star}$ (i)
}

\begin{abstract}
${ }^{1}$ Department of Neuroscience (DNS), University of Padova, Padua, Italy; ${ }^{2}$ International Consortium for Mood \& Psychotic Disorders Research, McLean Hospital, Belmont, Massachusetts, USA; ${ }^{3}$ Department of Psychiatry, Harvard Medical School, Boston, Massachusetts, USA and ${ }^{4}$ Lucio Bini Mood Disorders Centers, Cagliari, Italy
\end{abstract}

\begin{abstract}
Background. Ratings of affective temperament types show promise in helping to differentiate diagnostic groups among major affective disorders as well as to predict associations with important aspects of morbidity including suicidal risk.

Methods. The Temperament Evaluation of Memphis, Pisa, Paris, and San Diego auto-rating (TEMPS-A) questionnaire was completed by 858 unselected, consecutive, consenting adults diagnosed with a DSM-5 major affective disorder (173 bipolar-1 [BD-1]), 250 BD-2, 435 major depressive disorder [MDD]) to score for anxious $(a n x)$, cyclothymic $(c y c)$, dysthymic $(d y s)$, hyperthymic ( $h y p$ ), and irritable (irr) affective temperaments. We tested their associations with diagnosis and selected clinical factors, including diagnosis, depression scores, suicidal ideation or acts, substance abuse, episodes/year, and \%-time ill.

Results. Scores for cyc ranked: BD-2 > BD-1 > MDD; anx ranked: MDD > BD-2 > BD-1; irr was greater in $\mathrm{BD}$ than $\mathrm{MDD}$; dys was greater in $\mathrm{MDD}$ than $\mathrm{BD}$; hyp did not differ by diagnosis. We confirmed associations of suicidal risk with higher scores of all temperament types except lower hyp scores. Higher $c y c$ and irr scores and lower $a n x$ scores were associated with substance abuse. Several scores were higher with measures of greater affective morbidity: $c y c$ with current depression, episodes/year, and \%-time ill; irr with more episodes and depressions/year and greater \%-time manic. Some of these associations were selective for BD or MDD.

Conclusions. The findings indicate that TEMPS-A ratings of affective temperament types can contribute to differential diagnoses and predict types and amounts of affective morbidity, as well as detecting suicidal risks.
\end{abstract}

\section{Introduction}

Much more timely differentiation of major depressive (MDD), bipolar-1 (BD-1), and bipolar-2 (BD-2) disorders is clinically crucial to improving long-term planning aimed at better care of mood disorder patients [1]. Notably, the latency from initial clinical manifestations to a firm diagnosis and appropriate treatment of $\mathrm{BD}$ as distinct from unipolar depression averages 5-10 years, and even longer with onset in juvenile years, owing in large part to an excess of depression early in the course of $\mathrm{BD}[2,3]$. In striking contrast, nearly half of lifetime risk of suicidal acts (attempts and suicides) occurs within the first 2-3 years of these illnesses, and indeed such acts make early diagnosis more likely [4]. The uncertain differentiation of BD from MDD is underscored by the finding that more than half of patients originally diagnosed with a depressive episode eventually meet diagnostic criteria for BD, often owing to missing diagnosis of BD-2 through failure to recognize hypomania [5]. In addition, prolonged duration of untreated illness in $\mathrm{BD}$ is associated with more suicide attempts, greater affective and behavioral instability, and possibly more prolonged future illness $[6,7]$.

A potential contribution to improving early recognition of $\mathrm{BD}$ and MDD might include use of ratings of affective temperament or assessment of other aspects of temperament and personality, seeking potential links between a biological disposition to mood disorder and its clinical manifestations and considering some temperaments as antecedents of particular mood disorders [8,9]. Such assessments often rely on questionnaire-based rating schemes, including the Temperament Evaluation of Memphis, Pisa, Paris, and San Diego (TEMPS, often as a self- or auto-rating, TEMPS-A) [10], the Temperament and Character Inventory (TCI) [11], and the Neuroticism, Extraversion, Openness, Agreeableness, and Conscientiousness Scale (NEO-PI-3) [12]. In addition to supporting earlier, accurate diagnosis, such assessments might also have predictive value for the types and relative amounts of particular psychopathological features [13], and temperaments have shown strong relationships with suicidal behavior or ideation [14]. 
Several studies have focused on temperament assessments in individuals diagnosed with MDD [15-17] or with BD specifically [18-24], or have considered mood disorders together using TCI $[25,26]$ or TEMPS [1,27-30]. Such studies have revealed differences between mood disorder patients and controls, including unaffected family members, with suggestive differences between BD and MDD patients and possibly between $\mathrm{BD}-1$ and $\mathrm{BD}-2$ patients based on ratings of cyclothymic, hyperthymic, and irritable temperaments, in particular, as well as ratings of harm-avoidance [1,31-33]. There are also preliminary suggestions that temperament assessments may help to predict responses to antidepressant or mood-stabilizing treatments [28].

However, most studies focusing on assessment of temperament in individuals with major mood disorders suffer from various limitations. These include small sample size, data not systematically analyzed by multivariate analyses, as well as retrospective or crosssectional study designs that limit ability to form hypotheses regarding causality. In addition, it is important to consider potential effects of current mental state, which may influence responses to questions aimed at evaluating affective temperament [34]. Finally, relationships among affective temperament, morbidity indices, and clinical course in mood disorder patients have not been investigated extensively.

Given this background, the aim of the present study was to compare temperament profiles, assessed with the TEMPS-A questionnaire, in BD-1, BD-2 and MDD patient-subjects, and to test whether such assessments can contribute to differentiating among these diagnoses and can provide predictive associations with types or amounts of selected aspects of psychopathology.

\section{Methods}

\section{Study subjects}

Participants were adults evaluated and followed by the same mood disorder expert (LT) for several years at the Lucio Bini Mood Disorders Center in Cagliari, Sardinia, a specialized, academic outpatient clinic for the diagnosis, treatment, and study of affective disorder patients. They were consecutive and unselected except for adult age, presence of a DSM-5 major affective disorder (BD-1 or BD-2, MDD), and having completed TEMPS-A assessment. All were treated clinically and followed prospectively and systematically over several years. Written, informed consent was provided by all participants for collection and analysis of clinical data to be presented anonymously in aggregate form, following procedures approved by a local ethical review committee in accordance with requirements of Italian law and with the Helsinki Declaration. Study data were collected and entered a computerized database in coded form.

\section{Measures}

Current and lifetime diagnosis, course of illness, and psychiatric comorbidities were assessed according to DSM-5 diagnostic criteria [35]. TEMPS-A assessments were obtained following intake and initial treatment. Temperament was rated with the 39-item version of the self-rated TEMPS-A scale [36]. We considered raw, average numerical scores for five individual temperaments (cyclothymic $[c y c], 12$ items; dysthymic [dys], irritable [irr], hyperthymic [hyp], 8 items each; and anxious [anx], 3 items). Clinical measures were: diagnosis (BD-1, BD-2, MDD) score for the Hamilton Depression Rating Scale $\left(\mathrm{HDRS}_{21}\right)$, any suicidality (ideation or acts), or suicidal acts, substance abuse, episodes/year, and \%-time ill overall or in depression or [hypo]mania or total. For multivariate modeling, factors with preliminary bivariate differences were included stepwise as covariates.

\section{Data analysis}

Data are presented as means \pm standard deviation or with $95 \%$ confidence intervals (CI). Differences in TEMPS-A scores, sociodemographic factors, and morbidity indices were evaluated using contingency tables $\left(\chi^{2}\right)$ for categorical variables or analysis of variance ( $t$-test) for continuous data, followed by post hoc comparisons, or with bivariate linear regression $(r)$ to compare continuous measures. Statistics arising from preliminary bivariate comparisons were used to guide selection of factors to include in multivariable modeling, so as to limit effects of multiple comparisons. Statistics provided in tables are not repeated in the text. Analyses employed commercial software: Statview.5 (SAS Institute, Cary, NC) for spreadsheets, and Stata.13 (StataCorp, College Station, TX) for analyses.

\section{Results}

\section{Subject characteristics}

A total of 858 adults included: BD $(n=423)$, type $1(n=173)$ or $2(n=250)$, or MDD $(n=435)$. They were treated clinically and followed prospectively and systematically over an average of 7.99 [7.19-8.79] years. Age at intake averaged 46.2 [45.7-46.7] years; $62.9 \%$ [61.4-64.3] of subjects were women.

\section{TEMPS-A subscores versus diagnosis}

We compared scores for the five temperament types with three diagnoses (Table 1). Diagnostic subgroups differed highly significantly in TEMPS-A ratings of cyclothymic (cyc) and anxious (anx) temperament ( $p<0.001$ overall for both). The $c y c$ ratings ranked among the diagnoses as: BD- $>$ BD- $1>$ MDD; anx, ratings ranked: $\mathrm{MDD}>\mathrm{BD}-2>\mathrm{BD}-1$. Also significant were ratings for irritable temperament (irr) which ranked: BD-2 $\geq \mathrm{BD}-1>\mathrm{MDD}$ and for dysthymic temperament scores (dys), ranking: $\mathrm{MDD}>\mathrm{BD}-2>\mathrm{BD}-1$ ( $p=0.01$ overall for both). Ratings of hyperthymia (hyp) did not differ significantly among the diagnoses $(p=0.10)$.

In addition, the ratio of individual scores for cyc/anx strongly differentiated BD (3.89 [3.55-4.23]) from MDD (2.70 [2.48-2.92]) patients $(t=5.94, p<0.0001)$, whereas this ratio was very similar in BD-1 (3.83 [3.28-4.38]) and BD-2 (3.93 [3.50-4.36]) cases. The preceding findings suggest that high ratings for $c y c$ and low scores for $a n x$ may help to differentiate $\mathrm{BD}$ from $\mathrm{MDD}$, with additional contributions by high irr scores and low $d y s$ ratings also favoring BD over MDD.

\section{Clinical features associated with temperament ratings}

Among all 858 mood disorder participants, several clinically important features were associated with particular temperament ratings, irrespective of diagnosis (Table 2). Ratings of cyclothymic temperament $(c y c)$ were strongly associated with: alcohol abuse and substance abuse of any kind, as well as suicidal ideation or acts and suicidal acts (attempts and suicides) specifically. These scores were also significantly correlated with higher depression ratings at intake $\left(\mathrm{HDRS}_{21}\right)$, a greater proportion of time ill during 
Table 1. TEMPS-A temperament scores versus diagnosis.

\begin{tabular}{|c|c|c|c|c|c|}
\hline \multirow[b]{2}{*}{ Temperament } & \multicolumn{3}{|c|}{ Diagnosis (mean score $[95 \% \mathrm{Cl}]$ ) } & \multirow[b]{2}{*}{$t$-Score } & \multirow[b]{2}{*}{$p$-Value } \\
\hline & $\mathrm{BD}-1(n=173)$ & $\mathrm{BD}-2(n=250)$ & $\operatorname{MDD}(n=435)$ & & \\
\hline Cyclothymic & $5.32[4.83-5.81]$ & $5.91[5.51-6.31]$ & $4.80[4.51-5.09]$ & 31.7 & $<0.0001^{a}$ \\
\hline Anxious & $1.24[1.06-1.42]$ & $1.40[1.25-1.55]$ & $1.64[1.51-1.77]$ & 2.82 & $0.0004^{b}$ \\
\hline Irritable & $1.79[1.52-2.07]$ & $1.88[1.62-2.13]$ & $1.47[1.31-1.64]$ & 2.09 & $0.01^{c}$ \\
\hline Dysthymic & $3.22[2.91-3.53]$ & 3.56 [3.29-3.83] & $3.77[3.57-3.96]$ & 2.07 & $0.01^{d}$ \\
\hline Hyperthymic & $3.69[3.35-4.02]$ & $3.65[3.39-3.92]$ & $3.35[3.15-3.55]$ & 1.52 & 0.10 \\
\hline
\end{tabular}

Note: Temperaments are ranked by significance of diagnostic differences. Significant $(p \leq 0.05)$ post hoc comparisons: ${ }^{\mathrm{a} B D}-2>\mathrm{BD}-1>\mathrm{MDD} ;{ }^{\mathrm{b}} \mathrm{MDD}>\mathrm{BD}-2>\mathrm{BD}-1 ;{ }^{\mathrm{c}} \mathrm{BD}-2=\mathrm{BD}-1>\mathrm{MDD} ;{ }^{\mathrm{d}} \mathrm{MDD}>\mathrm{BD}-$ $2>$ BD-1. Scores vary with the number of items for each temperament type (e.g., 12 for cyclothymic and 3 for anxious).

Abbreviations: BD-1, type I bipolar disorder; BD-2, type II bipolar disorder; MDD, major depressive disorder.

Table 2. Clinical factors associated with TEMPS-A temperament assessment scores for 858 patients diagnosed with a DSM-5 major mood disorder.

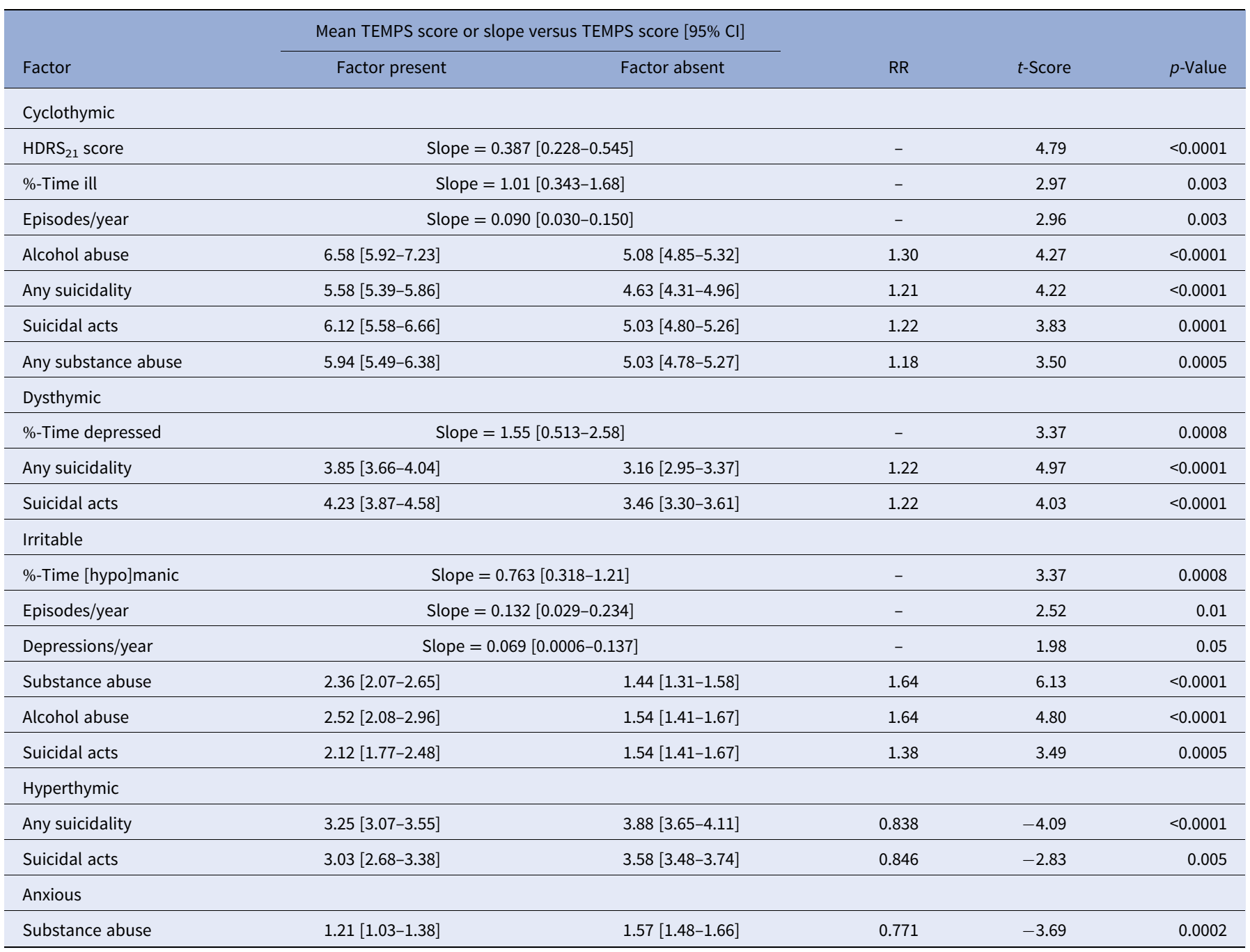

Note: Clinical factors were assessed during an average of 7.99 [7.19-8.79] years of prospective follow-up. All morbidity indices assessed were: depressions, [hypo]manias, or total episodes per year; \% time depressed, manic or total; $\mathrm{HDRS}_{21}$ score; any suicidality (acts + ideation), suicidal acts, substance abuse, and alcohol abuse.

several years of follow-up, and with a higher recurrence frequency (episodes/year).

Dysthymic temperament ratings (dys) were also highly significantly greater with both suicidal acts or any form of suicidality (including ideation and acts), and correlated strongly with higher percentage of time depressed.

Irritable temperament scores (irr) were highly significantly greater in patients with co-occurring abuse of any substances or of alcohol specifically, and with suicidal acts, and significantly correlated with more depressions/year, episodes/year, and especially with \%-of-time during follow-up in mania or hypomania (“[hypo]mania”).

As expected [14], scores for hyperthymic temperament (hyp) were highly significantly lower (by 15-16\%) in patients with a history of suicidal acts or with any suicidality (ideation or acts). 
Ratings of anxious temperament ( $a n x)$ were found to be significantly lower (by 23\%) in patients with co-occurring substance abuse than among those without substance abuse (Table 2).

\section{Diagnostic types associated with temperament ratings}

Some of the preceding findings among all mood disorder subjects were found selectively in $\mathrm{BD}$ or MDD, or occurred with both diagnoses (Table 3). Higher intake HDRS ${ }_{21}$ scores were strongly associated with higher scores for cyclothymic temperament $(c y c)$ in both BD and MDD patients, as well as with any lifetime suicidal ideation or acts, but with suicidal acts only among BD patients. Other clinical factors also were associated with higher $c y c$ scores selectively with $\mathrm{BD}$ but not MDD, notably including abuse of alcohol or of any substance, as well as \%-time-ill and episodes/year.

Ratings of dysthymic temperament $(d y s)$ were associated with $\%$-time-depressed and with suicidal acts or any suicidality in both $\mathrm{BD}$ and MDD patients.

Ratings of irritable temperament (irr) were elevated with cooccurring substance abuse in both BD and MDD patients. However, the following factors were associated with higher irr scores only with $\mathrm{BD}$ : alcohol abuse specifically, suicidal acts, and \%-time [hypo]manic, whereas depressions/year and mood episodes/year were not significantly associated with irr scores in either BD or MDD patients.

Hyperthymic temperament ratings ( $h y p$ ) were selectively lower with suicidal acts only among $\mathrm{BD}$, but not MDD patients. However, any suicidality, including suicidal ideation as well as suicidal acts, was associated with lower hyp scores in both diagnostic groups.

Lower ratings of anxious temperament (anx) were associated with substance abuse only among BD patients and not with MDD (Table 3).

\section{Multivariable regression modeling}

Based on the preliminary findings just summarized, we constructed multivariable logistic regression models for associations of temperament ratings with diagnosis (BD vs. MDD), substance abuse, and suicidal acts. In addition, we used linear regression models for $\%$-time during prospective follow-up in [hypo] mania or depression (Table 4). Diagnosis of BD was more likely than MDD with increased ratings for $c y c$, lower scores for anx and $d y s$, and lower ratings for irr. In addition, a particularly strong differentiating factor was the ratio of cyc/anx ratings. In logistic regression

Table 3. Clinical factors associated with TEMPS-A temperament assessment subscores with diagnoses of bipolar disorder versus major depressive disorder.

\begin{tabular}{|c|c|c|c|c|c|c|c|c|}
\hline \multirow[b]{3}{*}{ Factor } & \multicolumn{8}{|c|}{ Mean TEMPS score or slope versus TEMPS score $[95 \% \mathrm{CI}]$} \\
\hline & \multicolumn{4}{|c|}{ Bipolar disorder } & \multicolumn{4}{|c|}{ Major depression } \\
\hline & Factor present & Factor absent & $t$-Score & $p$-Value & Factor present & Factor absent & $t$-Score & $p$-Value \\
\hline \multicolumn{9}{|l|}{ Cyclothymic } \\
\hline HDRS $_{21}$ score & \multicolumn{2}{|c|}{ Slope $=0.490[0.254-0.727]$} & 4.01 & $<0.0001$ & \multicolumn{2}{|c|}{ Slope $=0.349[0.137-0.561]$} & 3.24 & 0.001 \\
\hline$\%$-Time ill & \multicolumn{2}{|c|}{ Slope $=1.20[0.323-2.09]$} & 2.69 & 0.008 & \multicolumn{2}{|c|}{ Slope $=0.04[-0.96$ to 1.04$]$} & 0.07 & 0.94 \\
\hline Episodes/year & \multicolumn{2}{|c|}{ Slope $=0.116[0.028-0.204]$} & 2.59 & 0.01 & \multicolumn{2}{|c|}{ Slope $=-0.008[-0.071$ to 0.054$]$} & 0.27 & 0.79 \\
\hline Any substance abuse & $6.73[5.99-7.47]$ & $5.48[5.13-5.84]$ & 2.95 & 0.003 & $6.00[3.79-8.21]$ & $4.74[4.44-5.04]$ & 1.80 & 0.07 \\
\hline Alcohol abuse & $6.73[5.99-7.47]$ & $5.48[5.13-5.84]$ & 2.95 & 0.003 & $6.00[4.51-7.49]$ & $4.74[4.44-5.04]$ & 1.80 & 0.07 \\
\hline Suicidal acts & $6.39[5.72-7.06]$ & $5.45[5.10-5.80]$ & 2.53 & 0.01 & $5.54[4.60-6.49]$ & $4.66[4.35-4.97]$ & 1.87 & 0.06 \\
\hline Any suicidality & $5.95[5.57-6.34]$ & $5.10[4.59-5.62]$ & 2.52 & 0.01 & $5.14[4.74-5.53]$ & $4.27[3.70-4.85]$ & 2.92 & 0.004 \\
\hline \multicolumn{9}{|l|}{ Dysthymic } \\
\hline$\%$-Time depressed & \multicolumn{2}{|c|}{ Slope $=1.55$ [0.513-2.58] } & 2.94 & 0.003 & \multicolumn{2}{|c|}{ Slope $=1.50[0.05-2.95]$} & 2.05 & 0.04 \\
\hline Suicidal acts & $4.19[3.76-4.62]$ & $3.20[297-3.44]$. & 4.07 & $<0.0001$ & $4.30[3.65-4.96]$ & $3.68[3.47-3.89]$ & 1.96 & 0.05 \\
\hline Any suicidality & 3.63 [3.37-3.89] & $3.05[2.73-3.37]$ & 2.59 & 0.01 & $4.12[3.86-4.39]$ & $3.24[2.96-3.53]$ & 4.39 & $<0.0001$ \\
\hline \multicolumn{9}{|l|}{ Irritable } \\
\hline$\%$-Time[hypo]manic & \multicolumn{2}{|c|}{ Slope $=0.773[0.123-1.42]$} & 2.34 & 0.02 & \multicolumn{2}{|c|}{ Slope $=0.026[-0.040$ to 0.100$]$} & 0.74 & 0.46 \\
\hline Episodes/year & \multicolumn{2}{|c|}{ Slope $=0.103[-0.042$ to 0.249$]$} & 1.40 & 0.16 & \multicolumn{2}{|c|}{ Slope $=0.107[-0.006$ to 0.220$]$} & 1.87 & 0.06 \\
\hline Depressions/year & \multicolumn{2}{|c|}{ Slope $=0.038[-0.049$ to 0.125$]$} & 0.85 & 0.39 & \multicolumn{2}{|c|}{ Slope $=0.098[-0.015$ to 0.210$]$} & 1.72 & 0.09 \\
\hline Any substance abuse & $2.51[2.15-2.88]$ & $1.58[1.37-1.80]$ & 4.56 & $<0.0001$ & $2.02[1.56-2.48]$ & 1.32 [1.15-1.50] & 2.92 & 0.004 \\
\hline Alcohol abuse & $2.63[2.13-3.14]$ & $1.72[1.52-1.93]$ & 3.56 & 0.0004 & $2.11[1.16-3.05]$ & 1.38 [1.21-1.55] & 1.84 & 0.07 \\
\hline Suicidal acts & $2.27[1.81-2.72]$ & $1.72[1.52-1.92]$ & 2.45 & 0.02 & $1.80[1.25-2.36]$ & 1.38 [1.21-1.55] & 1.59 & 0.11 \\
\hline \multicolumn{9}{|l|}{ Hyperthymic } \\
\hline Suicidal acts & 3.07 [2.63-3.51] & 3.83 [3.59-4.07]] & -3.06 & 0.002 & $2.96[2.37-3.55$ & 3.37 [3.54-3.58] & -1.29 & 0.20 \\
\hline Any suicidality & $3.45[3.18-3.71]$ & $4.07[3.72-4.42]$ & -2.80 & 0.007 & $3.04[2.77-3.30]$ & $3.73[3.42-4.04]$ & -3.23 & 0.0008 \\
\hline \multicolumn{9}{|l|}{ Anxious } \\
\hline Substance abuse & $1.11[0.90-1.31]$ & $1.43[1.24-1.63]$ & 2.63 & 0.009 & 1.43 [1.09-1.77] & $1.68[1.56-1.81]$ & 1.49 & 0.14 \\
\hline
\end{tabular}


modeling, along with lower $d y s$ and higher irr scores, the ratio of cyc/anx scores separated BD from MDD very strongly $(\mathrm{OR}=1.22$ $\left.[1.14-1.32], \chi^{2}=28.8, p<0.0001\right)$. The proportion of time in [hypo] mania was associated with decreased anx, and increased $c y c$, hyp, and irr scores. In contrast, the \%-time in depression was strongly and selectively associated only with higher $d y s$ scores. Substance abuse was associated strongly with increased irr scores, lower anx scores, and with BD more than MDD. Finally, suicidal acts (attempts and suicides) were strongly associated with BD > MDD and with higher $d y s$ ratings, and less strongly with increased irr and lower hyp scores (Table 4).

\section{Discussion}

This study involved data collected longitudinally for an average of 7.99 years of prospective observations of 858 unselected, consecutive individuals (a total of 6,855 person-years). We aimed to compare ratings of affective temperaments in patients diagnosed with a DSM-5 major affective disorder. We found several relationships of clinical interest that differentiated individuals with BD versus MDD and provided predictive associations with other clinical features of interest (Tables 3 and 4). This appears to be the first such study with a large sample size and longitudinal design aimed at evaluating the

Table 4. Multivariable models for clinical outcomes.

\begin{tabular}{|c|c|c|c|}
\hline Factor & OR or Slope $(\beta)[95 \% \mathrm{Cl}]$ & $\chi^{2}$ or $t$-Score & $p$-Value \\
\hline \multicolumn{4}{|c|}{ Diagnosis: $\mathrm{BD}>\mathrm{MDD}^{\mathrm{a}}$} \\
\hline Higher cyc & $1.14[1.09-1.20]$ & 25.4 & $<0.0001$ \\
\hline Lower anx & $0.775[0.686-0.875]$ & 16.9 & $<0.0001$ \\
\hline Lower dys & $0.859[0.797-0.926]$ & 15.6 & $<0.0001$ \\
\hline Higher irr & $1.10[1.01-1.19]$ & 4.97 & 0.029 \\
\hline \multicolumn{4}{|c|}{$\%$-Time [Hypo]manic ${ }^{b}$} \\
\hline Lower anx & $-0.875[-1.58$ to -0.169$]$ & 2.43 & 0.015 \\
\hline Higher cyc & $0.325[0.045-0.606]$ & 2.28 & 0.023 \\
\hline Higher hyp & $0.442[0.053-0.831]$ & 2.23 & 0.026 \\
\hline Higher irr & $0.517[0.039-0.995]$ & 2.12 & 0.034 \\
\hline \multicolumn{4}{|c|}{$\%$-Time depressed ${ }^{\mathrm{b}}$} \\
\hline Higher dys & $1.49[0.670-2.31]$ & 3.57 & $<0.0001$ \\
\hline \multicolumn{4}{|c|}{ Substance abuse ${ }^{a}$} \\
\hline Higher irr & $1.30[1.20-1.43]$ & 36.5 & $<0.0001$ \\
\hline $\mathrm{BD}>\mathrm{MDD}$ & $2.26[1.60-3.20]$ & 21.2 & $<0.0001$ \\
\hline Lower anx & $0.766[0.662-0.887]$ & 12.7 & 0.0004 \\
\hline \multicolumn{4}{|c|}{ Suicidal acts ${ }^{a}$} \\
\hline $\mathrm{BD}>\mathrm{MDD}$ & $2.49[1.70-3.64]$ & 22.0 & $<0.0001$ \\
\hline Higher dys & 1.14 [1.04-1.25] & 7.62 & 0.006 \\
\hline Lower hyp & $0.893[0.816-0.977]$ & 6.13 & 0.013 \\
\hline Higher irr & $1.13[1.02-1.24]$ & 5.94 & 0.015 \\
\hline
\end{tabular}

Note: Lower hyp scores with suicidal acts or ideation were found in preliminary bivariate analyses with both $B D$ and $M D D$ subjects, but selectively with $B D$ for suicidal acts (Table 3). Abbreviations: anx, anxious temperament; BD, bipolar disorder; cyc, cyclothymic temperament; dys, dysthymic temperament; hyp, hyperthymic temperament; irr, irritable temperament; MDD, major depressive disorder.

aLogistic regression modeling (OR)

Linear regression modeling (slope). predictive value of affective temperament ratings with diagnostic and morbidity measures in patients diagnosed reliably with DSM-5 BD-1, BD-2, or MDD.

Multivariable logistic regression modeling revealed that higher scores for cyclothymic and irritable temperaments were independently more likely among BD than MDD patients, whereas $d y s$ thymic and anxious temperament scores were higher in MDD than BD (Table 4). Moreover, the ratio of relatively high ratings for cyclothymic and low scores for anxious temperament was especially elevated with BD and distinguished BD from MDD.

Such results are consistent with previous studies comparing affective temperaments in patients with major mood disorders, in which $\mathrm{BD}$ cases showed higher cyclothymic and hyperthymic and lower anxious temperament scores than did MDD cases [27]. In addition, cyclothymic and hyperthymic temperament ratings have been reported to be $\mathrm{BD}$-selective [1]. We also found a preliminary association of $\mathrm{BD}$ diagnosis with elevated hyperthymia ratings (Table 1) that was not sustained in multivariable modeling (Table 4). Also of interest, relatives of $\mathrm{BD}$ patients have been reported to have higher cyclothymia scores than family members of MDD cases or healthy controls [27].

Consistent with our findings, Morishita et al. [29,30] found that cyclothymic and anxious temperament scores significantly differentiated the diagnosis of BD from MDD and statistically associated with $\mathrm{BD}$ by using multivariable logistic regression modeling. However, those studies also found that higher hyperthymic temperament scores differentiated subjects diagnosed with BD-1 versus BD-2, which we did not find (Table 1). The studies by Morishita et al. $[29,30]$ were based on a cross-sectional design and so may have missed some patients considered to have MDD who might later have met diagnostic criteria for BD [5,37,38]. Moreover, not all of their patients were currently in remission or euthymic, and some responses to temperament categorizing questions may have been influenced by current mood states [34,39].

Our findings confirmed associations of suicidal risk with higher scores of all temperament types except for hyperthymic, which were lower (Tables 3 and 4), as had been noted previously [14,40,41]. We also found that higher cyclothymic and irritable scores and lower anxious scores were associated with substance abuse. Though few previous studies focused on the temperament profile in BD patients with abuse of alcohol or other substances, in line with our findings (Table 3), cyclothymic or irritable temperament was reported to be associated with substance abuse (especially among BD patients) [42]. Low scores of anxious with high scores of irritable may reflect impulsivity commonly present with substance abuse. In addition, hyperthymia was associated with more severe hypomanic symptoms in multivariable modeling (Table 4), and in a previous study of 112 young adults at-risk for BD [42]. Cyclothymic and hyperthymic traits preceded abuse of stimulants by years, based on evaluating longitudinal progression of the dual pathology in a small sample of BD patients [43]. Among 1420 BD patients, several TEMPS-A scores were higher with alcohol abuse, particularly irritable and hyperthymic ratings, adjusted for potential confounders [19]. Finally, regression modeling based on 1,090 BD patients found abuse of alcohol and of other substances to be associated with irritable and hyperthymic temperaments, especially in males [21], whereas we found elevated scores of cyclothymic and irritable to be selectively associated with abuse of alcohol (Table 2).

We also found that several TEMPS-A scores were higher with measures of greater affective morbidity. In particular, higher ratings for dysthymia correlated strongly with the proportion of time in depression with both $\mathrm{BD}$ and MDD patients, whereas higher 
irritable ratings significantly correlated with more episodes/year and depressions/year, as well as with the proportion of time of BD subjects in mania or hypomania ("[hypo]mania"; Table 2). Finally, in addition to a strong association between cyclothymic temperament and initial depression severity assessed by $\mathrm{HDRS}_{21}$ (Table 2) in both MDD and BD subjects (Table 3), higher cyclothymia scores correlated significantly with \%-time-ill and episodes/year but selectively only among BD subjects (Table 3 ).

High cyclothymia scores seem to be associated with relatively unfavorable prognosis, perhaps as reflecting emotional and behavioral instability. This view is consistent with previous studies' finding that cyclothymic temperament can affect illness-course adversely [44-46].

Consistent with cyclothymic tendencies, mood reactivity and emotional dysregulation represent core psychopathological dimensions that often develop early in childhood [47]. BD patients, including those with cyclothymic temperament, are often initially misdiagnosed, typically as having MDD, potentially resulting in prolonged delay of appropriate treatment, higher rates of psychiatric comorbidity, and more histrionic, passive-aggressive, and less obsessive-compulsive personality types compared to those affected by $\mathrm{BD}$ without cyclothymic temperament-all probably tending to limit chances of attaining clinical remission $[47,48]$.

In a sample of 51 remitted BD subjects followed for 24 months, cyclothymic temperament scores were associated with greater overall functional impairment, including home management, and both individual and social leisure activities [45]. In line with the present study, with respect to BD patients (Table 3), high cyclothymia ratings have been reported to predict an excess of affective recurrences even when controlling for medication nonadherence [45]. Finally, cyclothymic temperament in BD patients has been associated with inferior treatment adherence and inferior response to medication $[20,49]$ as well as to psychoeducation [50].

\section{Limitations}

Some individuals may not have been fully euthymic, as their TEMPS-A assessments occurred early in their clinical assessment, soon after clinic entry, but none was acutely ill. Efforts to limit effects of multiple comparisons include the recommendation to consider of particular interest initial bivariate comparisons yielding $p$-value of $\leq 0.01$, and the limited statistics arising from multivariable modeling.

\section{Conclusions}

The present findings support the clinical value of rating affective temperament types, including to help differentiate BD from MDD diagnoses, limit risk of later changing diagnosis from MDD to BD, and to predict morbidity (mood states, recurrence rates, substance abuse, and suicidal risk), as well as highlighting the value of cyclothymic mood instability as a generally adverse prognostic indicator. The findings presented require replication and extension. Assessment of temperament is easily attained with the TEMPS-A scale, and should be considered as a component of routine evaluation of mood disorder patients, with possible particular value in the often difficult task of predicting a change of diagnosis from MDD to BD.

Data Availability Statement. The data that support the findings of this study are available from L.T. Restrictions are applied, given confidentiality issues.
Acknowledgments. R.J.B. was supported by a grant from the Bruce J. Anderson Foundation and by the McLean Private Donors Psychiatry Research Fund, and L.T. was supported by a grant from the Aretaeus Foundation of Rome.

Author Contributions. Conceptualization: A.M., M.P., L.T., R.J.B; Data curation: A.M., M.P., L.T.; Formal analysis: L.T., R.J.B; Investigation: L.T.; Methodology: A.M., M.P., L.T.; Writing_original draft: A.M., M.P., L.T., R.J.B; Writing-review and editing: L.T, R.J.B.

Conflicts of Interest. No author or immediate family member has financial relationships with commercial entities that might appear to represent potential conflicts of interest with the information presented.

\section{References}

[1] Serra G, Koukopoulos A, De Chiara L, Napoletano F, Koukopoulos AE, Curto $\mathrm{M}$, et al. Features preceding diagnosis of bipolar versus major depressive disorders. J Affect Disord. 2015;173:134-42. doi:10.1016/j. jad.2014.10.050.

[2] Baethge C, Tondo L, Bratti IM, Bschor T, Bauer M, Viguera AC, et al. Prophylaxis latency and outcome in bipolar disorders. Can J Psychiatry. 2003;48:449-57. doi:10.1177/070674370304800704.

[3] Baldessarini RJ, Tondo L, Baethge CJ, Lepri B, Bratti IM. Effects of treatment latency on response to maintenance treatment in manic-depressive disorders. Bipolar Disord. 2007;9:386-93. doi:10.1111/j.13995618.2007.00385.x.

[4] Tondo L, Baldessarini RJ, Hennen J, Floris G, Silvetti F, Tohen M. Lithium treatment and risk of suicidal behavior in bipolar disorder patients. J Clin Psychiatry. 1998;59:405-14. doi:10.4088/jcp.v59n0802.

[5] Angst J, Sellaro R, Stassen HH, Gamma A. Diagnostic conversion from depression to bipolar disorders: results of a long-term prospective study of hospital admissions. J Affect Disord. 2005;84:149-57. doi:10.1016/S01650327(03)00195-2.

[6] Altamura AC, Dell'Osso B, Berlin HA, Buoli M, Bassetti R, Mundo E. Duration of untreated illness and suicide in bipolar disorder: a naturalistic study. Eur Arch Psychiatry Clin Neurosci. 2010;260:385-91. doi:10.1007/ s00406-009-0085-2.

[7] Drancourt N, Etain B, Lajnef M, Henry C, Raust A, Cochet B, et al. Duration of untreated bipolar disorder: missed opportunities on the long road to optimal treatment. Acta Psychiatr Scand. 2013;127:136-44. doi: 10.1111/j.1600-0447.2012.01917.x.

[8] Gonda X, Fountoulakis KN, Juhasz G, Rihmer Z, Lazary J, Laszik A, et al. Association of the $s$ allele of the 5-HTTLPR with neuroticism-related traits and temperaments in a psychiatrically healthy population. Eur Arch Psychiatry Clin Neurosci. 2009;259:106-13. doi:10.1007/s00406-0080842-7.

[9] Gonda X, Eszlari N, Torok D, Gal Z, Bokor J, Millinghoffer A, et al. Genetic underpinnings of affective temperaments: a pilot GWAS investigation identifies a new genome-wide significant SNP for anxious temperament in ADGRB3 gene. Transl Psychiatry. 2021;11:337-400. doi:10.1038/ s41398-021-01436-1.

[10] Akiskal HS, Akiskal KK, Hayakal RF, Manning JS, Connor PD. TEMPS-A: progress towards validation of a self-rated clinical version of the Temperament Evaluation of the Memphis, Pisa, Paris, and San Diego Autoquestionnaire. J Affect Disord. 2005;85:3-16. doi:10.1016/j. jad.2004.12.001.

[11] Cloninger CR, Svrakic DM, Przybeck TR. A psychobiological model of temperament and character. Arch Gen Psychiatry. 1993;50:975-90. doi: 10.1001/archpsyc.1993.01820240059008.

[12] McCrae RR, Costa PT, Martin TA. NEO-PI-3, a more readable, revised NEO personality inventory. J Pers Assess. 2005;84:261-70. doi:10.1207/ s15327752jpa8403_05.

[13] Fountoulakis KN, Gonda X, Koufaki I, Hyphantis T, Cloninger CR. Role of temperament in the etiopathogenesis of bipolar spectrum illness. Harv Rev Psychiatry. 2016;24:36-52. doi:10.1097/HRP.0000000000000077.

[14] Vázquez GH, Gonda X, Lolich M, Tondo L, Baldessarini RJ. Suicidal risk and affective temperaments evaluated with TEMPS-A. Harv Rev Psychiatry. 2018;26:8-18. doi:10.1097/HRP.0000000000000153. 
[15] Maina G, Salvi V, Rosso G, Bogetto F. Cyclothymic temperament and major depressive disorder: a study on Italian patients. J Affect Disord. 2010;12:199-203. doi:10.1016/j.jad.2009.05.031.

[16] Kudo Y, Nakagawa A, Wake T, Ishikawa N, Kurata C, Nakahara M, et al. Temperament, personality, and treatment outcome in major depression: a 6-month preliminary prospective study. Neuropsychiatr Dis Treat. 2017; 13:17-24. doi:10.2147/NDT.S123788.

[17] Gurpegui D, Ortuño F, Gurpegui M. Temperament traits and remission of depression: a naturalistic six-month longitudinal study. J Affect Disord. 2019;243:494-502. doi:10.1016/j.jad.2018.09.041.

[18] Perugi G, Toni C, Maremmani I, Tusini G, Ramacciotti S, Madia A, et al. The influence of affective temperaments and psychopathological traits on the definition of bipolar disorder subtypes: a study on bipolar I Italian national sample. J Affect Disord. 2012;136:e41-9. doi:10.1016/j. jad.2009.12.027.

[19] Singh S, Forty L, di Florio A, Gordon-Smith K, Jones I, Craddock N, et al. Affective temperaments and concomitant alcohol use disorders in bipolar disorder. J Affect Disord. 2015;186:226-31. doi:10.1016/j.jad.2015.07.027.

[20] Buturak SV, Emel EB, Koçak OM. The effect of temperament on the treatment adherence of bipolar disorder type I. Nord J Psychiatry. 2016; 70:176-82. doi:10.3109/08039488.2015.1067330.

[21] Azorin J-M, Perret LC, Fakra E, Tassy S, Simon N, Adida M, et al. Alcohol use and bipolar disorders: risk factors associated with their co-occurrence and sequence of onsets. Drug Alcohol Depend. 2017;179:205-12. doi: 10.1016/j.drugalcdep.2017.07.005.

[22] Perugi G, Cesari D, Vannucchi G, Maccariello G, Barbuti M, De Bartolomeis A, et al. Impact of affective temperaments on clinical and functional outcome of bipolar I patients that initiated or changed pharmacological treatment for mania. Psychiatry Res. 2018;261:473-80. doi:10.1016/j.psychres.2018.01.035.

[23] Altınbaş K, İnce B, Gülöksüz S. Affective temperament and seasonality in bipolar disorder. Psychiatr Danub. 2019;31:106-10. doi:10.24869/ psyd.2019.106.

[24] Fico G, Caivano V, Zinno F, Carfagno M, Steardo LJ, Sampogna G, et al. Affective temperaments and clinical course of bipolar disorder: exploratory study of differences among patients with and without a history of violent suicide attempts. Med Kaunas Lith. 2019;55:E390-401. doi: 10.3390/medicina55070390.

[25] Zaninotto L, Souery D, Calati R, Di Nicola M, Montgomery S, Kasper S, et al. Temperament and character profiles in bipolar I, bipolar II and major depressive disorder: impact over illness course, comorbidity pattern and psychopathological features of depression. J Affect Disord. 2015;184:51-9. doi:10.1016/j.jad.2015.05.036.

[26] Balestri M, Porcelli S, Souery D, Kasper S, Dikeos D, Ferentinos P, et al. Temperament and character influence on depression treatment outcome. J Affect Disord. 2019;252:464-74. doi:10.1016/j.jad.2019.04.031.

[27] Aguiar Ferreira AD, Vasconcelos AG, Neves FS, Laks J, Correa H. Affective temperaments: familiality and clinical use in mood disorders. J Affect Disord. 2013;148:53-6. doi:10.1016/j.jad.2012.11.047.

[28] Aguiar Ferreira AD, Vasconcelos AG, Neves FS, Correa H. Affective temperaments and antidepressant response in the clinical management of mood disorders. J Affect Disord. 2014;155:138-41. doi:10.1016/j. jad.2013.10.038.

[29] Morishita C, Kameyama R, Toda H, Masuya J, Ichiki M, Kusumi I, et al. Utility of TEMPS-A in differentiation between major depressive disorder, bipolar I disorder, and bipolar II disorder. PLoS One. 2020;15: e0232459-68. doi:10.1371/journal.pone.0232459.

[30] Morishita C, Kameyama R, Toda H, Masuya J, Fujimura Y, Higashi S, et al. TEMPS-A (short version) plays a supplementary role in the differential diagnosis between major depressive disorder and bipolar disorder. Psychiatry Clin Neurosci. 2021;75:166-71. doi:10.1111/pcn.13198.

[31] Loftus ST, Garno JL, Jaeger J, Malhotra AK. Temperament and character dimensions in bipolar I disorder: comparison to healthy controls. J Psychiatr Res. 2008;42:1131-6. doi:10.1016/j.jpsychires.2007.11.005.

[32] Solmi M, Zaninotto L, Toffanin T, Veronese N, Lin K, Stubbs B, et al. Comparative meta-analysis of TEMPS scores across mood disorder patients, their first-degree relatives, healthy controls, and other psychiatric disorders. J Affect Disord. 2016;196:32-6. doi:10.1016/j. jad.2016.02.013.
[33] Zaninotto L, Solmi M, Toffanin T, Veronese N, Cloninger CR, Correll CU. Meta-analysis of temperament and character dimensions in patients with mood disorders: comparison to healthy controls and unaffected siblings. J Affect Disord. 2016;194:84-97. doi:10.1016/j.jad.2015.12.077.

[34] Baba H, Kohno K, Inoue T, Nakai Y, Toyomaki A, Suzuki T, et al. The effects of mental state on assessment of bipolar temperament. J Affect Disord. 2014;161:1-3. doi:10.1016/j.jad.2014.03.004.

[35] American Psychiatric Association (APA). Diagnostic and statistical manual of mental disorders (DSM-5). 5th ed. Arlington, VA: American Psychiatric Publishing; 2013.

[36] Akiskal HS, Mendlowicz MV, Jean-Louis G, Rapaport MH, Kelsoe JR, Gillin JC, et al. TEMPS-A: validation of a short version of a self-rated instrument designed to measure variations in temperament. J Affect Disord. 2005;85:45-52. doi:10.1016/j.jad.2003.10.012.

[37] Dudek D, Siwek M, Zielińska D, Jaeschke R, Rybakowski J. Diagnostic conversions from major depressive disorder into bipolar disorder in an outpatient setting: results of a retrospective chart review. J Affect Disord. 2013;144:112-5. doi:10.1016/j.jad.2012.06.014.

[38] Kim EY, Kim NW, Kim MJ, Yang BR, Rhee SJ, Park CHK, et al. Rate of diagnostic conversion to bipolar disorder in adults with unipolar depression and psychopharmacological treatment in the republic of Korea: nationwide register-based study. J Affect Disord. 2020;273:240-6. doi: 10.1016/j.jad.2020.03.159.

[39] Morvan Y, Tibaoui F, Bourdel MC, Lôo H, Akiskal KK, Akiskal HS, et al. Confirmation of the factorial structure of temperamental autoquestionnaire TEMPS-A in non-clinical young adults and relation to current state of anxiety, depression and to schizotypal traits. J Affect Disord. 2011;131: 37-44. doi:10.1016/j.jad.2011.01.008.

[40] Pompili M, Baldessarini RJ, Innamorati M, Vázquez GH, Rihmer Z, Gonda X, et al. Temperaments in psychotic and major affective disorders. J Affect Disord. 2018;225:195-200. doi:10.1016/j.jad.2017.08.026.

[41] Tondo L, Vázquez GH, Sani G, Pinna M, Baldessarini RJ. Association of suicidal risk with ratings of affective temperaments. J Affect Disord. 2018; 229:322-7. doi:10.1016/j.jad.2017.12.061.

[42] DeGeorge DP, Walsh MA, Barrantes-Vidal N, Kwapil TR. A three-year longitudinal study of affective temperaments and risk for psychopathology. J Affect Disord. 2014;164:94-100. doi:10.1016/j.jad.2014.04.006.

[43] Camacho A, Akiskal HS. Proposal for a bipolar-stimulant spectrum: temperament, diagnostic validation and therapeutic outcomes with mood stabilizers. J Affect Disord. 2005;85:217-30. doi:10.1016/j. jad.2003.10.014.

[44] Mechri A, Kerkeni N, Touati I, Bacha M, Gassab L. Association between cyclothymic temperament and clinical predictors of bipolarity in recurrent depressive patients. J Affect Disord. 2011;132:285-8. doi:10.1016/j. jad.2011.02.006.

[45] Nilsson KK, Straarup KN, Jørgensen CR, Licht RW. Affective temperaments' relation to functional impairment and affective recurrences in bipolar disorder patients. J Affect Disord. 2012;138:332-6. doi:10.1016/j. jad.2012.01.020.

[46] Innamorati M, Rihmer Z, Akiskal H, Gonda X, Erbuto D, Belvederi Murri $\mathrm{M}$, et al. Cyclothymic temperament rather than polarity is associated with hopelessness and suicidality in hospitalized patients with mood disorders. J Affect Disord. 2015;170:161-5. doi:10.1016/j.jad.2014.08.042.

[47] Perugi G, Hantouche E, Vannucchi G. Diagnosis and treatment of cyclothymia: the "primacy" of temperament. Curr Neuropharmacol. 2017;15: 372-9. doi:10.2174/1570159X14666160616120157.

[48] Akiskal HS, Hantouche EG, Allilaire JF. Bipolar II with and without cyclothymic temperament: "dark" and "sunny" expressions of soft bipolarity. J Affect Disord. 2003;73:49-7. doi:10.1016/s0165-0327(02)00320-8.

[49] Fornaro M, De Berardis D, Iasevoli F, Pistorio ML, D’Angelo E, Mungo S, et al. Treatment adherence towards prescribed medications in bipolar-II acutely depressed patients: relationship with cyclothymic temperament and "therapeutic sensation seeking" in response towards subjective intolerance to pain. J Affect Disord. 2013;151:596-604. doi:10.1016/j. jad.2013.07.004.

[50] Reinares M, Pacchiarotti I, Solé B, García-Estela A, Rosa AR, Bonnín CM, et al. A prospective longitudinal study searching for predictors of response to group psychoeducation in bipolar disorder. J Affect Disord. 2020;274: 1113-21. doi:10.1016/j.jad.2020.02.047. 